\title{
Inflation-Produced Magnetic Fields in $R^{n} F^{2}$ and $I F^{2}$ models
}

\author{
L. Campanelli ${ }^{1,2}$ * P. Cea ${ }^{1,2}$ 丹 G.L. Fogli ${ }^{1,2}$ 团 and L. Tedesco ${ }^{1,2 \S}$ \\ ${ }^{1}$ Dipartimento di Fisica, Università di Bari, I-70126 Bari, Italy and \\ ${ }^{2}$ INFN - Sezione di Bari, I-70126 Bari, Italy
}

(Dated: February, 2008)

\begin{abstract}
We re-analyze the production of seed magnetic fields during Inflation in $\left(R / m^{2}\right)^{n} F_{\mu \nu} F^{\mu \nu}$ and $I F_{\mu \nu} F^{\mu \nu}$ models, where $n$ is a positive integer, $R$ the Ricci scalar, $m$ a mass parameter, and $I \propto \eta^{\alpha}$ a power-law function of the conformal time $\eta$, with $\alpha$ a positive real number. If $m$ is the electron mass, the produced fields are uninterestingly small for all $n$. Taking $m$ as a free parameter we find that, for $n \geq 2$, the produced magnetic fields can be sufficiently strong in order to seed dynamo mechanism and then to explain galactic magnetism. For $\alpha \gtrsim 2$, there is always a window in the parameters defining Inflation such that the generated magnetic fields are astrophysically interesting. Moreover, if Inflation is (almost) de Sitter and the produced fields almost scale-invariant $(\alpha \simeq 4)$, their intensity can be strong enough to directly explain the presence of microgauss galactic magnetic fields.
\end{abstract}

PACS numbers: 98.62.En, 98.80.-k

\section{INTRODUCTION}

Microgauss magnetic fields are present in any type of galaxies [1]. Up to now, astronomical observations have not been able to clarify the nature and origin of these fields, so that "galactic magnetism" is still an open issue in cosmology.

If on the one hand, the presence of magnetic fields in all galaxies could be explained by the action of astrophysical mechanisms operating during or after large-scale structure formation [2], on the other hand, the detection of magnetic fields in galaxies at high redshifts [3] represent a strong hint that magnetic fields could have been generated in the very early Universe. Clearly, the detection of magnetic fields in the Cosmic Microwave Background radiation would prove the primordial origin of cosmic magnetic fields [4]. In such a case, it is very probable that they have been generated during an inflationary epoch of the Universe, since only in this case it is possible to generate fields correlated on comoving scale as large as galactic ones.

Indeed, many mechanisms [5, 6, 7, 8, 9], acting during Inflation, have been proposed to produce seed fields since the seminal paper by Turner and Widrow [10].

It is worth noting that, in order to explain galactic magnetism, one needs the presence of seed magnetic fields prior to galaxy formation of intensity generally much less than $1 \mu \mathrm{G}$. In fact, when a protogalaxy collapses to form a galactic disk, magnetic fields suffer an amplification due to magnetic flux conservation. Moreover, due to magnetohydrodynamic turbulence effects and differential rotation of galaxy, seed fields can be further amplified. This last mechanism, know as "galactic dynamo" [11], can be very efficient and, in principle, allow extremely week seeds to reproduce the properties of presently-observed galactic fields.

If dynamo is operating, the present-day intensity and correlation length required for a successful amplification are ${ }^{1} B \gtrsim 10^{-33} \mathrm{G}$ and $\lambda \gtrsim 10 \mathrm{kpc}$ while, without dynamo, a comoving seed field $B \gtrsim 10^{-14} \mathrm{G}$ correlated on comoving scales of order $1 \mathrm{Mpc}$ is needed to explain galactic magnetism [5]. Note that, following Ref. [1], we are assuming that the redshift at which a protogalaxy separates from the Hubble flow to then collapse is $z_{\mathrm{pg}} \simeq 50$.

In this paper, we re-analyze the generation of seed magnetic fields during Inflation considering non-standard terms in the electromagnetic Lagrangian of the form $\left(R / m^{2}\right)^{n} F_{\mu \nu} F^{\mu \nu}$ and $I F_{\mu \nu} F^{\mu \nu}$, where $n$ is a positive integer, $R$ the Ricci scalar, $m$ a mass parameter, and $I(\phi, R, \ldots)$ a scalar function depending on any scalar field, gravity, and on any other background field.

The first model was first proposed by Turner and Widrow [10] for the case $n=1$ and then extended to the case $n \geq 1$ by Mazzitelli and Spedalieri [6]. They found that, taking $m=m_{e}$ ( $m_{e}$ being the electron mass), astrophysically interesting seed fields can be generated during Inflation for adequate values of $n$. In Section II, we will find a different result from Ref. [6]. Indeed, if $m=m_{e}$, the produced fields are uninterestingly small for all $n$. Nevertheless, taking $m$ as a free parameter, the possibility to generate magnetic fields sufficiently strong in order to seed dynamo mechanism is not excluded.

The second model was first investigated by Bamba and Sasaki [7] who assumed de Sitter Inflation and $I$ to be a power-law function of the conformal time $\eta, I \propto \eta^{\alpha}$, with $\alpha$ a negative real number. In Section III, we will extend this model to the case of positive real $\alpha$ for both Power-Law and de Sitter Inflation.

\footnotetext{
${ }^{1}$ It is worth noting that galactic dynamo is a still-debated and model-dependent amplification mechanism, and that the minimum value of the seed field used in this paper, $B \simeq 10^{-33} \mathrm{G}$, corresponds indeed to an extremely efficient dynamo action. For details see, e.g., Ref. [5] and references therein.
} 
In Section IV we draw our conclusions.

\section{II. $R^{n} F^{2}$ MODELS}

We consider a photon $A_{\mu}$ described by the effective Lagrangian

$$
\mathcal{L}=-\frac{1}{4} F_{\mu \nu} F^{\mu \nu}-\frac{1}{4} I F_{\mu \nu} F^{\mu \nu}, \quad I=\left(\frac{R}{m^{2}}\right)^{n},
$$

where $F_{\mu \nu}=\partial_{\mu} A_{\nu}-\partial_{\nu} A_{\mu}$ is the electromagnetic field strength tensor, $R$ the Ricci scalar, $m$ a parameter having dimension of a mass, and $n$ a positive integer. (From now on, Greek indices range from 0 to 3, while Latin ones from 1 to 3 .)

We will work in a flat Universe described by a Robertson-Walker metric $d s^{2}=a^{2}\left(d \eta^{2}-d \mathbf{x}^{2}\right)$, where the conformal time $\eta$ is related to the cosmic time $t$ through $d \eta=d t / a$. We normalize the expansion parameter so that at the present time $t_{0}, a\left(t_{0}\right)=1$.

We consider the case of Power-Law Inflation described by the equation of state $p_{\text {tot }}=\gamma \rho_{\text {tot }}$ with $-1<\gamma<-1 / 3$. In this case, the Hubble parameter $H^{2}=(8 \pi / 3) \rho_{\mathrm{tot}} / m_{\mathrm{Pl}}^{2}$ and the Ricci scalar $R=$ $8 \pi(1-3 \gamma) \rho_{\text {tot }} / m_{\text {Pl }}^{2}$ evolve as $H^{2} \propto R \propto \rho_{\text {tot }} \propto \eta^{s}$, where $s=-6(1+\gamma) /(1+3 \gamma)$. Here, $\rho_{\text {tot }}\left(p_{\text {tot }}\right)$ is the total energy (pressure) density during Inflation and $m_{\mathrm{Pl}} \simeq 1.22 \times 10^{19} \mathrm{GeV}$ is the Planck mass.

We assume that during Inflation $R \gg m^{2}$, so that we can neglect the Maxwell term in Lagrangian (11). Since $R$ is a decreasing function of time, it suffices to impose that $R_{1} \lesssim m^{2}$ or equivalently $m \lesssim m_{\max }$, with

$$
\frac{m_{\max }}{m_{\mathrm{Pl}}}=\sqrt{8 \pi(1-3 \gamma)}\left(\frac{M}{m_{\mathrm{Pl}}}\right)^{2},
$$

where $M^{4}=\rho_{\text {tot }}\left(\eta_{1}\right)$ is the total energy density at the end of Inflation. Here and in the following, the subscript "1" indicates the time when Inflation ends.

The spectrum of gravitational waves generated at Inflation is submitted to constraints coming from Cosmic Microwave Background analysis which requires $\rho_{\text {tot }}$ to be less than about $10^{-8} \mathrm{~m}_{\mathrm{Pl}}^{4}$ on the scale of the present Hubble radius [10]. This turns in a upper limit on the value of $M$ and $s: M \lesssim 10^{-2} m_{\mathrm{Pl}}$ and $s \lesssim s_{\max }$, where [10] $s_{\max } \simeq-\left[8+4 \log _{10}\left(M / m_{\mathrm{Pl}}\right)\right] /\left[29.8+\log _{10}\left(M / m_{\mathrm{Pl}}\right)\right]$.

One must also impose that $M \gtrsim 1 \mathrm{GeV}$, so that the predictions of Big Bang Nucleosynthesis are not spoiled [10].

We work in the Coulomb gauge, $A_{0}=\sum_{i} \partial_{i} A_{i}=0$, and we expand the electromagnetic field $A_{\mu}=\left(A_{0}, \mathbf{A}\right)$ as

$$
\mathbf{A}(\eta, \mathbf{x})=\int \frac{d^{3} k}{(2 \pi)^{3} \sqrt{2 k}} \sum_{\alpha=1,2} \varepsilon_{\mathbf{k}, \alpha} a_{\mathbf{k}, \alpha} A_{k}(\eta) e^{i \mathbf{k x}}+\text { h.c. },
$$

where $k=|\mathbf{k}|$ and $\varepsilon_{\mathbf{k}, \alpha}$ are the transverse polarization vectors satisfying the completeness relation $\sum_{\alpha}\left(\varepsilon_{\mathbf{k}, \alpha}\right)_{i}\left(\varepsilon_{\mathbf{k}, \alpha}^{*}\right)_{j}=P_{i j}(\mathbf{k})$, with $P_{i j}(\mathbf{k})=\delta_{i j}-k_{i} k_{j} / k^{2}$. In order that the annihilation and creation operators $a_{\mathbf{k}, \alpha}$ and $a_{\mathbf{k}, \alpha}^{\dagger}$ satisfy the usual commutation relations $\left[a_{\mathbf{k}, \alpha}, a_{\mathbf{k}^{\prime}, \alpha^{\prime}}^{\dagger}\right]=(2 \pi)^{3} \delta_{\alpha \alpha^{\prime}} \delta\left(\mathbf{k}-\mathbf{k}^{\prime}\right)$, we must impose the normalization condition $A_{k}(\eta) \dot{A}_{k}^{*}(\eta)-A_{k}^{*}(\eta) \dot{A}_{k}(\eta)=i / I$, where a dot denotes differentiation with respect to $\eta$. This assures, in turn, that the field $A_{\mu}$ and its canonical conjugate momenta $\pi_{0}=0$ and $\pi_{i}=I \dot{A}_{i}$ satisfy the usual commutation relations:

$$
\left[A_{i}(\eta, \mathbf{x}), \pi_{j}(\eta, \mathbf{y})\right]=i \int \frac{d^{3} k}{(2 \pi)^{3}} e^{i \mathbf{k}(\mathbf{x}-\mathbf{y})} P_{i j}(\mathbf{k}) .
$$

The equation of motion for $A_{k}(\eta)$ follows from Lagrangian (11):

$$
\ddot{A}_{k}(\eta)+\frac{n s}{\eta} \dot{A}_{k}(\eta)+k^{2} A_{k}(\eta)=0 .
$$

The solution of the above equation, taking into account the normalization condition on $A_{k}(\eta)$, is easily found:

$$
A_{k}(\eta)=\sqrt{\frac{\pi}{2}} e^{-i \pi(1+2 \nu) / 4} I^{-1 / 2}|k \eta|^{1 / 2} H_{\nu}^{(2)}(|k \eta|),
$$

where $\nu=(n s-1) / 2$ and $H_{\nu}^{(2)}(x)$ is the Hankel function of second kind. (Observe that, since both $n$ and $s$ are positive, it results $\nu \geq-1 / 2$ ).

We are interested in the study of large-scale electromagnetic fields, that is in modes whose physical wavelength is much greater than the Hubble radius, $\lambda_{\text {phys }} \gg H^{-1}$ or equivalently $|k \eta| \ll 2 /|1+3 \gamma|$, where $\lambda_{\text {phys }}=a \lambda, \lambda=1 / k$ is the comoving wavelength, and we used $\eta=2 /[(1+3 \gamma) a H]$. Therefore, in Eq. (6), we can replace the Hankel function with its smallargument expansion: $H_{\nu}^{(2)}(x) \simeq i \pi^{-1} 2^{\nu} \Gamma(\nu) x^{-\nu}$ for $\nu>0, H_{\nu}^{(2)}(x) \simeq 2 i \pi^{-1} \ln x$ for $\nu=0$, and $H_{\nu}^{(2)}(x) \simeq$ $-i \pi^{-1} 2^{-\nu} \Gamma(-\nu) e^{-i \pi \nu} x^{\nu}$ for $\nu<0$, where $\Gamma(x)$ is the Gamma function.

After Inflation, the Universe enters in the so-called reheating phase, during which the energy of the inflaton is converted into ordinary matter. In this paper, we restrict our analysis to the case of instantaneous reheating, that is after Inflation the Universe enters the radiation dominated era. In radiation era, the general expression for the electromagnetic field is

$$
A_{k}^{\mathrm{rad}}(\eta)=\alpha_{k} e^{i k \eta}+\beta_{k} e^{-i k \eta},
$$

where $\alpha_{k}$ and $\beta_{k}$ are the so-called Bogoliubov coefficients [12], determining the spectral number distribution of particles produced from the vacuum. By matching expressions (6) and (7) and their first derivative at the time of the end of Inflation, we find the spectrum of the 
electromagnetic field generated from the vacuum at large scales:

$$
\left|A_{k}^{\mathrm{vac}}\left(\eta_{1}\right)\right|^{2}=\left|\beta_{k}\right|^{2} \simeq \frac{\left[2^{\nu} \Gamma(1+\nu)\right]^{2}}{2 \pi I_{1}\left|k \eta_{1}\right|^{1+2 \nu}},
$$

valid for $\nu \geq 0$. When $-1 / 2<\nu<0$, the electromagnetic vacuum fluctuations go like $\left|A_{k}^{\mathrm{vac}}\left(\eta_{1}\right)\right|^{2} \propto\left|k \eta_{1}\right|^{1+2 \nu}$ and then are vanishingly small for $\left|k \eta_{1}\right| \ll 1$. In the case $\nu=-1 / 2$, conformal invariance is recovered and then, as it should be, we find $\left|A_{k}^{\mathrm{vac}}\left(\eta_{1}\right)\right|^{2}=0$, exactly, for all $\left|k \eta_{1}\right|$.

We can define, now, the average magnetic field on a comoving scale $\lambda$ as [9]

$$
B_{\lambda}^{2}(\eta)=a^{-4}\left\langle 0|| \int d^{3} y W_{\lambda}(|\mathbf{x}-\mathbf{y}|) \nabla \times\left.\mathbf{A}(\eta, \mathbf{y})\right|^{2} \mid 0\right\rangle,
$$

where $W_{\lambda}(|\mathbf{x}|)$ is a suitable (real) window function, and the vacuum state $|0\rangle$, such that $a_{\mathbf{k}, \alpha}|0\rangle=0$, is normalized as $\langle 0 \mid 0\rangle=1$. Taking into account Eqs. (3) and (9), we obtain

$$
B_{\lambda}^{2}(\eta)=\int_{0}^{\infty} \frac{d k}{k} W_{\lambda}^{2}(k) \mathcal{P}_{B}(\eta, k),
$$

where $W_{\lambda}(k)$ is the Fourier Transform of the window function and

$$
\mathcal{P}_{B}(\eta, k)=\frac{k^{4}}{2 \pi^{2} a^{4}}\left|A_{k}(\eta)\right|^{2}
$$

is the magnetic power spectrum. As windows function we can take a gaussian window, $W_{\lambda}(|\mathbf{x}|)=$ $\left(2 \pi \lambda^{2}\right)^{-3 / 2} e^{-|\mathbf{x}|^{2} /\left(2 \lambda^{2}\right)}$, so that $W_{\lambda}(k)=e^{-\lambda^{2} k^{2} / 2}$.

From the end of Inflation until today, due to the high conductivity of the cosmic plasma, the magnetic field evolves adiabatically [10], $a^{2} B_{\lambda}=$ const, so that $B_{\lambda}^{\text {today }}=a_{1}^{2} B_{\lambda}\left(\eta_{1}\right)$. Inserting Eq. (8) in Eq. (11) and taking into account Eq. (10), we finally obtain:

$$
\begin{aligned}
& B_{\lambda}^{\text {today }}=\frac{2^{\nu} \Gamma(1+\nu)[\Gamma(3 / 2-\nu)]^{1 / 2}}{(2 \pi)^{3 / 2} I_{1}^{1 / 2}} \frac{\lambda^{\nu-3 / 2}}{\left|\eta_{1}\right|^{\nu+1 / 2}}, \\
& I_{1}=\left(\frac{m_{\max }}{m}\right)^{2 n} \\
& \left|\eta_{1}\right|=\frac{\sqrt{3}}{\sqrt{2 \pi}|1+3 \gamma|}\left[\frac{g_{* S}\left(T_{1}\right)}{g_{* S}\left(T_{0}\right)}\right]^{1 / 3} \frac{m_{\mathrm{Pl}}}{M T_{0}} .
\end{aligned}
$$

In Eq. (14), we used the fact that during radiation and matter dominated eras the expansion parameter evolves as $a \propto g_{* S}^{-1 / 3} T^{-1}$, where $T$ is the temperature and $g_{* S}(T)$ the number of effectively massless degrees of freedom referring to the entropy density of the Universe [13]. The temperature at the end of Inflation, the so-called reheating temperature, is 10] $T_{1}=M$, while $T_{0}$ is the actual temperature. ${ }^{2}$

\footnotetext{
${ }^{2}$ In the following we will use the values [13]: $T_{0} \simeq 2.35 \times$
}
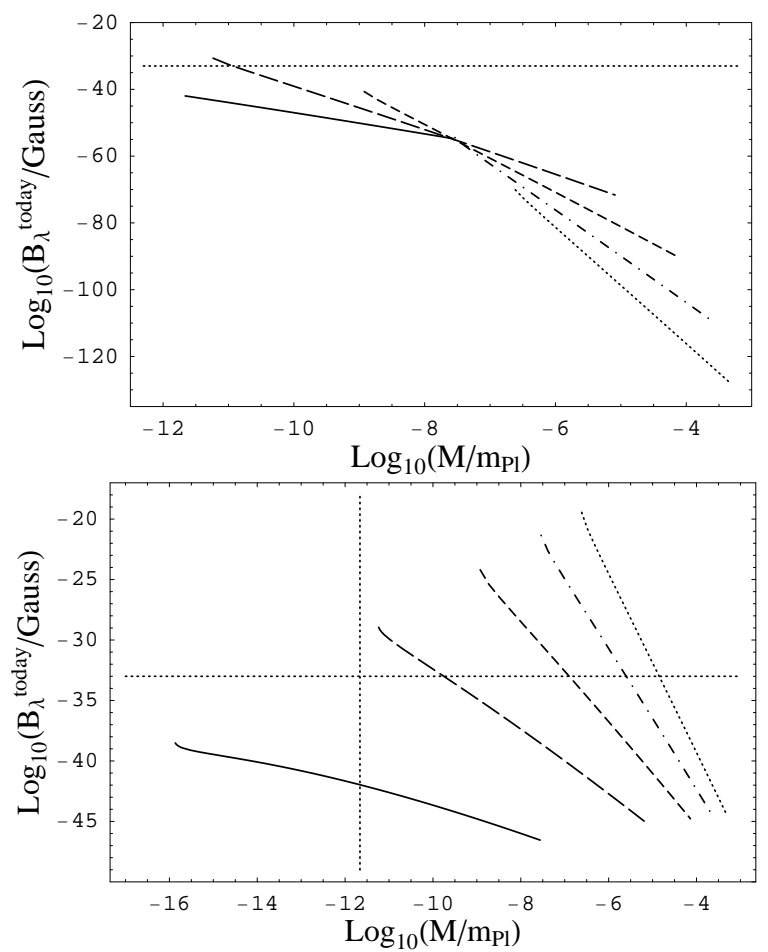

FIG. 1: Actual magnetic field as a function of $M$ for $m=m_{e}$ (upper panel) and $m=m_{\max }$ (lower panel) at the comoving scale $\lambda=10 \mathrm{kpc}$ for different values of $n$ : $n=1$ (continuous line), $n=2$ (long-dashed line), $n=3$ (dashed line), $n=4$ (dot-dashed line), $n=5$ (dotted line). The horizontal dotted lines refers to the minimum seed field required for dynamo amplification, $B \simeq 10^{-33} \mathrm{G}$. In the lower panel, on the left (right) side of the vertical dotted line it results $m \lesssim m_{e}(m \gtrsim$ $\left.m_{e}\right)$.

It is important to observe that, in order to avoid an infrared divergence in the magnetic power spectrum, we must impose that $\nu<3 / 2$.

The "best case scenario", i.e. the case of maximum strength of the magnetic field, corresponds to $s=s_{\max }$.

In Fig. 1, we show the actual magnetic field, in the best case scenario, as a function of the Inflation energy scale $M$ for different values of the index $n$ at the comoving scale $\lambda=10 \mathrm{kpc}$ in the two cases $m=m_{e}$ (upper panel) and $m=m_{\max }$ (lower panel). The range of $M$ is such that $0 \leq \nu \leq 1.49$ (both panels) and $m \lesssim m_{\max }$ (upper panel).

As it is clear from the figure, if $m=m_{e}$ the produced fields are not astrophysically interesting. This disagrees with the results discussed by Mazzitelli and Spedalieri in

$10^{-13} \mathrm{GeV}, g_{* S}\left(T_{0}\right) \simeq 3.91$, and $g_{* S}\left(T_{1}\right)=106.75$ (referring to the number of effectively massless degrees of freedom of Standard Model). It is useful to recall that $1 \mathrm{G} \simeq 6.9 \times 10^{-20} \mathrm{GeV}^{2}$ and $1 \mathrm{Mpc} \simeq 1.56 \times 10^{38} \mathrm{GeV}^{-1}$. 
Ref. [6] where it is argued that, for adequate values of $n$ and $\gamma$, the produced fields can serve as seed for the cosmological magnetic field. The discrepancy resides in the fact that, the astrophysically interesting fields they obtained correspond, indeed, to values of $n$ and $\gamma$ (and then of $\nu$ ) such that the related power spectrum is infrareddivergent, and then are non-physical.

In the "optimistic" case $m=m_{\max }$, for all $n \geq 2$ there is a range in the parameter $M$ such that the intensity of the produced magnetic field is greater than the minimum seed field required for dynamo amplification, $B \gtrsim 10^{-33} \mathrm{G}$.

\section{III. $I F^{2}$ MODELS}

We consider now a model given by Lagrangian (11) with $I$ an arbitrary power function of the conformal time:

$$
I=I_{1}\left(\frac{\eta}{\eta_{1}}\right)^{\alpha}
$$

where $I_{1}$ is a constant and we assume that $\alpha$ is a positive real number. The above parametrization of the function $I$, already used in the literature [7], is not the unique admissible one. However, leaving $I$ an arbitrary function of the conformal time, if on the one hand would render our analysis more general, on the other hand would allow us just to get qualitative results.

We study the production of seed fields during an inflationary epoch described by both Power-Law and de Sitter Inflation. In the latter case, the equation of state describing the evolution of the Universe is $p_{\text {tot }}=\gamma \rho_{\text {tot }}$ with $\gamma=-1$, so that the Hubble parameter is a constant and $\eta=-1 /(a H)$.

If $I_{1} \gtrsim 1$, we can neglect the Maxwell term in Eq. (1) and then the analysis performed in Section II applies also to the case at hand. In particular, Eqs. (15), (6), (12), and (14) are still valid provided $n s$ is replaced by $\alpha$. Consequently, now it results $\nu=(\alpha-1) / 2$ and the condition $0 \leq \nu<3 / 2$ translates to $1 \leq \alpha<4$. The expression for the actual magnetic field can be recast as:

$B_{\lambda}^{\text {today }}=\frac{10^{12 \alpha-55}}{\zeta_{\alpha} I_{1}^{1 / 2}} \frac{[\Gamma(2-\alpha / 2)]^{1 / 2}}{|1+3 \gamma|^{-\alpha / 2}}\left(\frac{M}{m_{\mathrm{Pl}}}\right)^{\alpha / 2} \lambda_{10 \mathrm{kpc}}^{(\alpha-4) / 2} \mathrm{G}$,

where $\lambda_{10 \mathrm{kpc}}=\lambda /(10 \mathrm{kpc})$ and $\zeta_{\alpha}$ is an increasing function of $\alpha$ of order unity ( $\operatorname{such}$ that $\zeta_{1} \simeq 0.6$ and $\zeta_{4} \simeq 2.3$ ).

In Fig. 2, we show the value of actual magnetic field both for Power-Law (in the best case scenario corresponding to $s=s_{\max }$ ) and de Sitter Inflation as a function of $M$ (which in this case can assume all values in the interval $1 \mathrm{GeV} \lesssim M \lesssim 10^{-2} m_{\mathrm{Pl}}$ ) for different values of the parameter $\alpha$ at the comoving scale $\lambda=10 \mathrm{kpc}$ and for $I_{1}=1$.

We find that for $\alpha \gtrsim 2$ there is always a minimum value of $M, M_{\min }$, such that if $M \gtrsim M_{\min }$ the generated

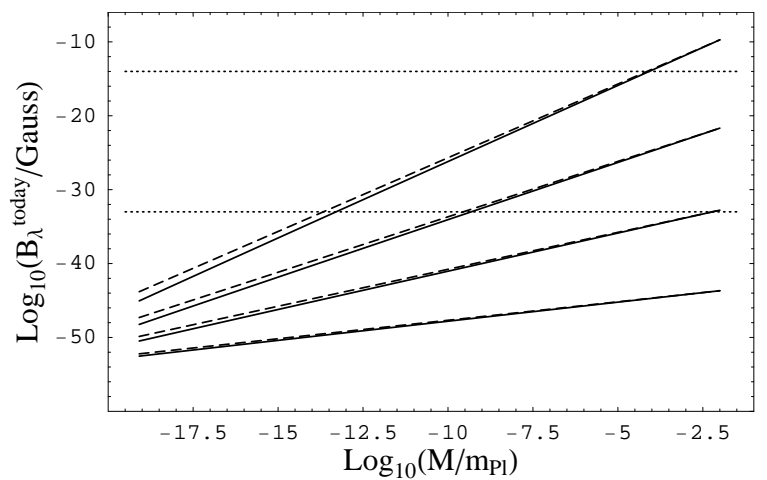

FIG. 2: Actual magnetic field in the case of Power-Law (continuous lines) and de Sitter (dashed lines) Inflation as a function of $M$ for different values of $\alpha$. From down to top: $\alpha=1,2,3,3.99$. The first two curves from the top correspond to $\lambda=1 \mathrm{Mpc}$, while the other ones to $\lambda=10 \mathrm{kpc}$. The horizontal dotted lines refer to the minimum seed fields required for dynamo amplification, $B \simeq 10^{-33} \mathrm{G}$, and to directly explain galactic magnetism, $B \simeq 10^{-14} \mathrm{G}$.

magnetic field is greater than the minimum seed field required for a successful dynamo amplification. Moreover, if the field is almost scale-invariant, i.e. $\alpha \simeq 4$, and if $M$ is large enough, its intensity can be strong enough to directly explain galactic magnetism (see the first two curves from the top in Fig. 2 referring to $\lambda=1 \mathrm{Mpc}$ ). In fact, expanding Eq. (16) about $\alpha=4$ and taking $\gamma \simeq-1$ (which corresponds to take large values of $M$ ), we find

$$
B_{\lambda}^{\text {today }} \simeq \frac{2 \times 10^{-7}}{\sqrt{1-\alpha / 4}}\left(\frac{M}{m_{\mathrm{Pl}}}\right)^{2} \mathrm{G},
$$

giving $B_{\lambda}^{\text {today }} \gtrsim 10^{-14} \mathrm{G}$ for $M \gtrsim 3(1-\alpha / 4)^{1 / 4} 10^{15} \mathrm{GeV}$.

\section{CONCLUSIONS}

Why are large-scale magnetic fields present in all galaxies is still a debated question in cosmology. They origin could reside in an amplification of Inflation-produced electromagnetic vacuum fluctuations through galacticdynamo mechanisms.

In this paper, we have re-analyzed the production of seed magnetic fields during Inflation considering notconformal-invariant terms in the electromagnetic Lagrangian of the form $\left(R / m^{2}\right)^{n} F_{\mu \nu} F^{\mu \nu}$ and $I F_{\mu \nu} F^{\mu \nu}$.

The first model was first proposed by Turner and Widrow [10] for the case $n=1$ and then extended to the case $n \geq 1$ by Mazzitelli and Spedalieri [6].

The second model was first investigated by Bamba and Sasaki 7] in the framework of de Sitter Inflation and assuming $I$ to be a power-law function of the conformal time $\eta, I \propto \eta^{\alpha}$, with $\alpha$ a negative real number.

Concerning the first model, Mazzitelli and Spedalieri [6] found that, taking $m=m_{e}\left(m_{e}\right.$ be- 
ing the electron mass), astrophysically interesting seed fields can be generated during Inflation for adequate values of $n$. Our analysis instead, as discussed in Section II, shows that if $m$ is the electron mass, the produced fields are uninterestingly small for all $n$. Nevertheless, taking $m$ as a free parameter, the possibility to generate magnetic fields sufficiently strong in order to seed dynamo mechanism is not excluded.

Regarding the second model, we have extended it to the case of positive real $\alpha$ for both Power-Law and de Sitter Inflation. We have found that, for $\alpha \gtrsim 2$, there is always a window in the parameters defining Inflation such that the generated magnetic fields are astrophysically interesting. Moreover, if Inflation is (almost) de Sitter and the produced fields almost scale-invariant $(\alpha \simeq 4)$, their intensity can be strong enough to directly explain the presence of microgauss galactic magnetic fields.

* Electronic address: leonardo.campanelli@ba.infn.it

$\dagger$ Electronic address: paolo.cea@ba.infn.it

¥ Electronic address: gianluigi.fogli@ba.infn.it

$\S$ Electronic address: luigi.tedesco@ba.infn.it

[1] For reviews on cosmic magnetic fields see: L. M. Widrow, Rev. Mod. Phys. 74, 775 (2002); M. Giovannini, Int. J. Mod. Phys. D 13, 391 (2004); D. Grasso and H. R. Rubinstein, Phys. Rept. 348, 163 (2001).

[2] R. E. Pudritz and J. Silk, Astrophys. J. 342, 650 (1989); A. Lazarian, Astron. Astrophys. 264, 326 (1992); K. Subramanian, D. Narasimha, and S. M. Chitre, MNRAS bf 271, L15 (1994); R. M. Kulsrud, et al., Astrophys. J. 480, 481 (1997); G. Davies and L. M. Widrow, ibid. 540, 755 (2000); N. Y. Gnedin, A. Ferrara, and E. G. Zweibel, ibid. 539, 505 (2000); E. R. Siegel and J. N. Fry, ibid. 651, 627 (2006).

[3] P. P. Kronberg, J. J. Perry, and E. L. H. Zukowski, Astrophys. J. 387, 528 (1992); A. M. Wolfe, K. M. Lanzetta, and A. L. Oren, ibid. 388, 17 (1992); R. M. Wolfe, et al., Astron. Astrophys. 329, 809 (1998).

[4] A. Kosowsky and A. Loeb, Astrophys. J. 469, 1 (1996); J. D. Barrow, P. G. Ferreira, and J. Silk, Phys. Rev. Lett. 78, 3610 (1997); K. Subramanian and J. D. Barrow, ibid. 81, 3575 (1998); R. Durrer, T. Kahniashvili, and A. Yates, Phys. Rev. D 58, 123004 (1998); R. Durrer, P. G. Ferreira, and T. Kahniashvili, ibid. 61, 043001 (2000); K. Jedamzik, V. Katalinic, and A. V. Olinto, Phys. Rev. Lett. 85, 700 (2000); T. R. Seshadri and K. Subramanian, ibid. 87, 101301 (2001); A. Mack, T. Kahniashvili, and A. Kosowsky, Phys. Rev. D 65, 123004 (2002); L. Campanelli, A. D. Dolgov, M. Giannotti, and F. L. Villante, Astrophys. J. 616, 1 (2004);
A. Lewis, Phys. Rev. D 70, 043011 (2004); M. Giovannini, ibid. 71, 021301 (2005); A. Kosowsky, et al., ibid. 71, 043006 (2005); J. D. Barrow, R. Maartens, and C. G. Tsagas, Phys. Rept. 449, 131 (2007), and references therein; L. Campanelli, P. Cea and L. Tedesco, Phys. Rev. Lett. 97, 131302 (2006) [Erratum-ibid. 97, 209903 (2006)]; Phys. Rev. D 76, 063007 (2007); M. Giovannini, ibid. 70, 123507 (2004); Class. Quant. Grav. 23, 4991 (2006); Phys. Rev. D 73, 101302 (2006); ibid. 74, 063002 (2006); PMC Phys. A 1, 5 (2007) arXiv:0706.4428 [astro-ph]]; Phys. Rev. D 76, 103508 (2007); D. G. Yamazaki, K. Ichiki, T. Kajino, and G. J. Mathews, Phys. Rev. D 77, 043005 (2008).

[5] L. Campanelli, P. Cea, G. L. Fogli, and L. Tedesco, Phys. Rev. D 77, 043001 (2008).

[6] F. D. Mazzitelli and F. M. Spedalieri, Phys. Rev. D 52, 6694 (1995).

[7] K. Bamba and M. Sasaki, JCAP 0702, 030 (2007).

[8] B. Ratra, Astrophys. J. 391, L1 (1992); W. D. Garretson, G. B. Field, and S. M. Carroll, Phys. Rev. D 46, 5346 (1992); A. Dolgov, ibid. 48, 2499 (1993); D. Lemoine and M. Lemoine, ibid. 52, 1955 (1995); M. Gasperini, M. Giovannini, and G. Veneziano, Phys. Rev. Lett. 75, 3796 (1995); A. C. Davis and K. Dimopoulos, Phys. Rev. D 55, 7398 (1997); O. Bertolami and D. F. Mota, Phys. Lett. B 455, 96 (1999); M. Giovannini, Phys. Rev. D 62, 123505 (2000); hep-ph/0104214 Phys. Rev. D 64, 061301 (2001); T. Prokopec, astro-ph/0106247; M. Gasperini, Phys. Rev. D 63, 047301 (2001); B. A. Bassett, G. Pollifrone, S. Tsujikawa and F. Viniegra, ibid. 63, 103515 (2001); K. Dimopoulos, T. Prokopec, O. Tornkvist, and A. C. Davis, ibid. 65, 063505 (2002); K. Bamba and J. Yokoyama, Phys. Rev. D 69, 043507 (2004); ibid. 70, 083508 (2004); A. Ashoorioon and R. B. Mann, ibid. 71, 103509 (2005); C. G. Tsagas, ibid. 72, 123509 (2005); K. E. Kunze, Phys. Lett. B 623, 1 (2005); M. R. Garousi, M. Sami and S. Tsujikawa, ibid. 606, 1 (2005); M. M. Anber and L. Sorbo, JCAP 0610, 018 (2006); A. AkhtariZavareh, A. Hojati, and B. Mirza, Prog. Theor. Phys. 117, 803 (2007); K. E. Kunze, arXiv:0710.2435 [astroph]; M. Giovannini, Phys. Lett. B 659, 661 (2008); J. Martin and J. Yokoyama, JCAP 0801, 025 (2008).

[9] T. Prokopec and E. Puchwein, Phys. Rev. D 70, 043004 (2004).

[10] M. S. Turner and L. M. Widrow, Phys. Rev. D 37, 2743 (1988).

[11] For recent reviews on dynamo mechanisms see, e.g.: A. Brandenburg and K. Subramanian, Phys. Rept. 417, 1 (2005); A. Shukurov, astro-ph/0411739.

[12] N. D. Birrell and P. C. W. Davies, Quantum Fields in Curved Space (Cambridge University Press, New York, 1982).

[13] E. W. Kolb and M. S. Turner, The Early Universe (Addison-Wesley, Redwood City, California, 1990). 